\title{
A high-resolution optofluidic microscope with optical tweezer actuation
}

Xin Heng, Edward Hsiao, Demetri Psaltis, Changhuei Yang

Xin Heng, Edward Hsiao, Demetri Psaltis, Changhuei Yang, "A highresolution optofluidic microscope with optical tweezer actuation," Proc. SPIE 6441, Imaging, Manipulation, and Analysis of Biomolecules, Cells, and Tissues V, 644113 (19 February 2007); doi: 10.1117/12.701728 


\title{
A High Resolution OptoFluidic Microscope with Optical Tweezer Actuation
}

\author{
Xin Heng ${ }^{1}$, Edward Hsiao ${ }^{1}$, Demetri Psaltis ${ }^{1}, 2$, and Changhuei Yang ${ }^{1}$ \\ ${ }^{1}$ Department of Electrical Engineering (MC136-93), California Institute of Technology, Pasadena, CA, USA; \\ ${ }^{2}$ School of Engineering, Ecole Polytechnique Federale de Lausanne, Switzerland. \\ xin@caltech.edu
}

\begin{abstract}
In this paper, we will report our recent development of a new type of OptoFluidic Microscope (OFM) that is capable of delivering resolution beyond the diffraction limit of light. Accurate control of the sample translation is accomplished by adopting an optical tweezer scanner into the system. During the image acquisition, a two-dimensional nanoaperture array defined on a thin aluminum film acts as an array of ultra-fine illumination sources. The imaging system is tested and demonstrated by using polystyrene beads and green algae (Chlamydomonas). Properties of the system are reported and discussed.
\end{abstract}

\section{Introduction}

Recently, there is a growing interest in investigating subwavelength apertures as they possess intriguing optical transmission and diffraction properties [1-4]. Subwavelength apertures are routinely used as imaging probes in commercial near field optical microscopes (NSOM), as these nanoaperture probes can deliver ultrahigh resolution beyond the diffraction limit. The resolution of such microscopes is in principle only limited by the size of the aperture. This idea of super resolution microscopy was first proposed by Synge, S. H. in 1928 [5], and it is only in the recent past that NSOM systems have started to be extensively used in research laboratories. NSOM has proven capable of imaging extremely fine structures of chemical, biological or environmental samples with resolution beyond the scope of conventional optical microscopes [6, 7].

However, NSOM is not a simple and cheap tool to operate. A commercial NSOM system usually requires a sophisticated feedback control system and an ultra stable two-dimension raster scanning stage during image acquisition. This requirement makes the whole NSOM unit slow and difficult to operate. Secondly, NSOM users have to spend a long time on prealigning the system and locating the objects of interest before really taking a high-resolution image of the samples. Therefore improvement of NSOM system for the ease of use is desirable

Along the line of creating cheap and compact high-resolution systems, our group lately reported a new type of imaging device, termed optofluidic microscope (OFM) [8]. The first OFM prototype used a one-dimension subwavelength aperture array to collect the light scattering from the biological samples. Time-of-flight signals recorded by each aperture were subsequently used to construct a picture of the sample. In order to eliminate under-sampling, the OFM employs a slanted nanoaperture array configuration. In addition, microfluidic control with pressure driven flow was employed to stably move the samples across the nanoaperture array. High throughput (40 samples/minute) and high resolution $(500 \mathrm{~nm})$ imaging of wild type $C$. elegans was successfully demonstrated.

In this paper, we present our recent development on a new type of OFM, where nanoapertures are used as illumination sources rather than collection units as in the first OFM prototype [8]. Furthermore, a 2D nanoaperture array replaced the 1D aperture array that was in the first OFM prototype, so that the length of the whole imaging region can be reduced by 5 to 10 folds depending on the size of the samples. In addition, a robust optical tweezer scanner 
is built and then utilized to stably translate the sample across the $2 \mathrm{D}$ nanoaperture region. It will be demonstrated that high resolution imaging $(\sim 100 \mathrm{~nm})$ is made possible by fusing OFM with optical tweezers.

\section{Experiment Methods}

Setup of the entire imaging system is presented in Figure 1(a). Ti-Sapphire laser (Spectral Physics Tsunami) operating at $800 \mathrm{~nm}$ in cw mode is the laser for the optical tweezer. The laser is coupled into a single-mode fiber, collimated and then steered by a computercontrolled PZT mirror (from Physik Instrumente). A 4-f lens relay is added into the scanning system, so that when the laser beam is steered by the PZT mirror, the center of the laser beam always hits the center of the back aperture of the high numerical aperture (NA) objective (Olympus, 40X (NA=0.8, water, IR). Note that this $4 \mathrm{f}$ relay technique has been routinely used in commercial laser scanning confocal microscopes. When the power of the trapping laser is sufficiently high, the sample will be held firmly within the tight focus of the objective lens. The power of the trapping laser is estimated to be $30 \mathrm{~mW}$, which proves to be safe enough for the biological samples used in this experiment. The light components of the trapping beam back-scattered from the nanoaperture plate are strong and can easily saturate the recording camera's CCD pixels. In order to avoid this saturation problem, a cold mirror and an IR filter pair are mounted in the system.

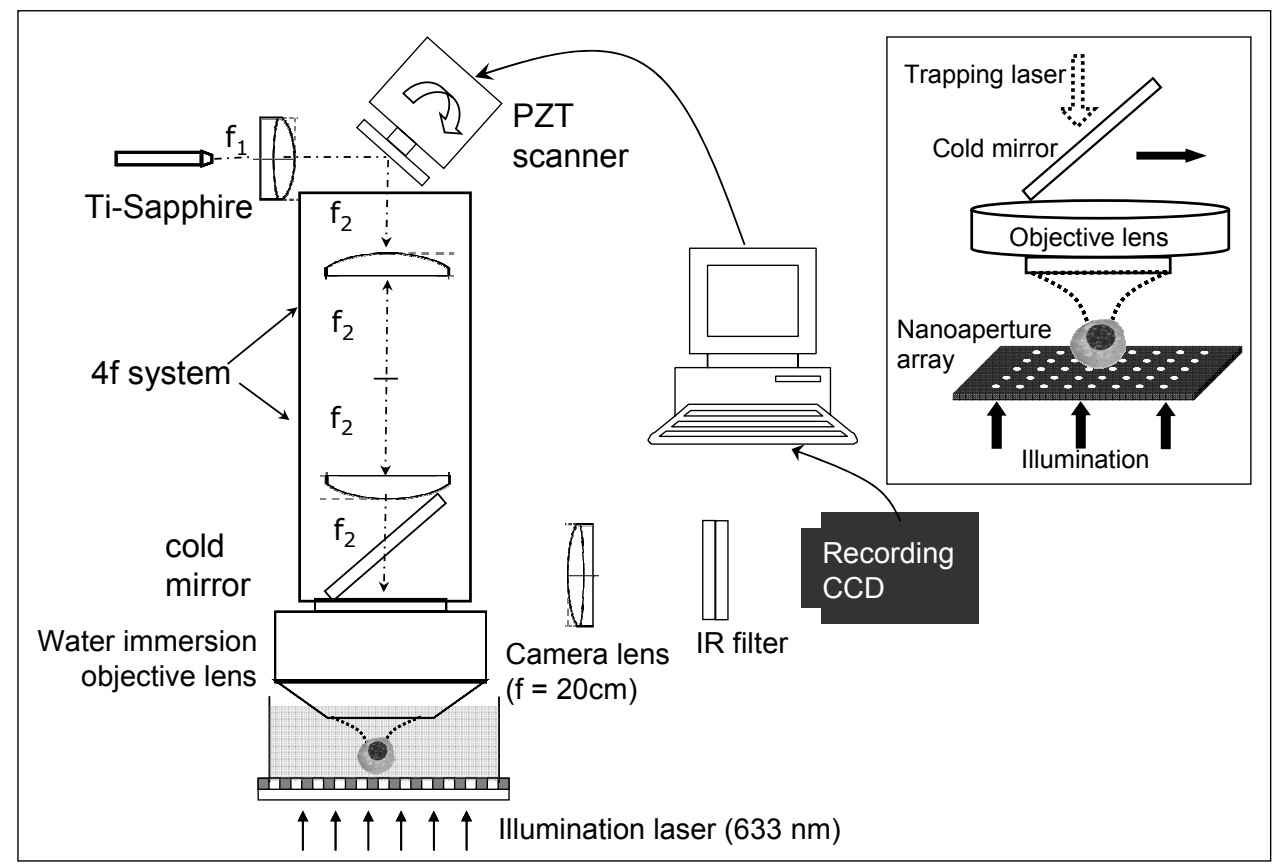

Figure 1: Setup of the imaging system. Ti-Sapphire: Ti-Sapphire laser (800 nm, $30 \mathrm{~mW})$. Water immersion objective lens: Olympus, 40X, NA=0.8, water, IR. Illumination laser: He$\mathrm{Ne}, 633 \mathrm{~nm}, 20 \mathrm{~mW}$. Recording CCD: Princeton Instruments (Spec10-100). Sample (only for demonstration purpose): nucleated red blood cell. (b) Zoom-in of Fig. 1 setup showing the 2D nanoaperture array and its arrangement with the optical tweezer. 
From below, a He-Ne laser $(633 \mathrm{~nm}, 20 \mathrm{~mW})$ provides illumination. The light transmitted through the nanoaperture array form a two-dimensional illumination grid. This illumination grid is nicely mapped onto the recording CCD camera (see Fig. 2(a)) through the use of the same objective lens and an achromatic camera lens $(\mathrm{f}=20.0 \mathrm{~cm})$. When a sample is passing by the nanoaperture region, it will scatter or absorb the illumination light, resulting in changes in brightness of the nanoapertures that are mapped onto the screen of the CCD camera. These time-of-light signal changes are later used to construct a sample image. Fig. 1(b) is a zoom-in of the optical setup, showing the 2D nanoaperture array and how it is arranged with the objective lens, i.e. the optical tweezer.

The operating principle of the imaging system can be explained with Fig. 2(b). $L_{x}\left(L_{y}\right)$ represents the hole spacing in $\mathrm{x}(\mathrm{y})$ direction. The sample tightly held by the optical trap is scanned across the nanoapertures at a tilt angle $(\theta)$ with respect to $\mathrm{x}$ axis. During the image formation, the pixel size in $\mathrm{y}$ direction $(\delta y)$ and that in $\mathrm{x}$ direction $(\delta x)$ are expressed as following:

$$
\begin{aligned}
& \delta y=L_{x} \sin (\theta) \\
& \delta x=V \delta t
\end{aligned}
$$

where $V$ is the velocity of the sample and $\delta t$ is the camera speed. All the parameters mentioned above can be accurately defined by fabrication, optical alignment and the computer-controlled PZT-scanner. Note that the sample passes over each of the apertures at different time. For example, hole $\alpha$ and hole $\beta$ in Fig. 2(b), although scan adjacent parts of the sample, will 'see' this object with a time delay between them. Therefore, a distorted image will be formed if we directly use the time traces of the nanoapertures. However, as the moving speed of the sample and the camera speed are constant, the time-delay factors between different holes have a simple linear relationship.

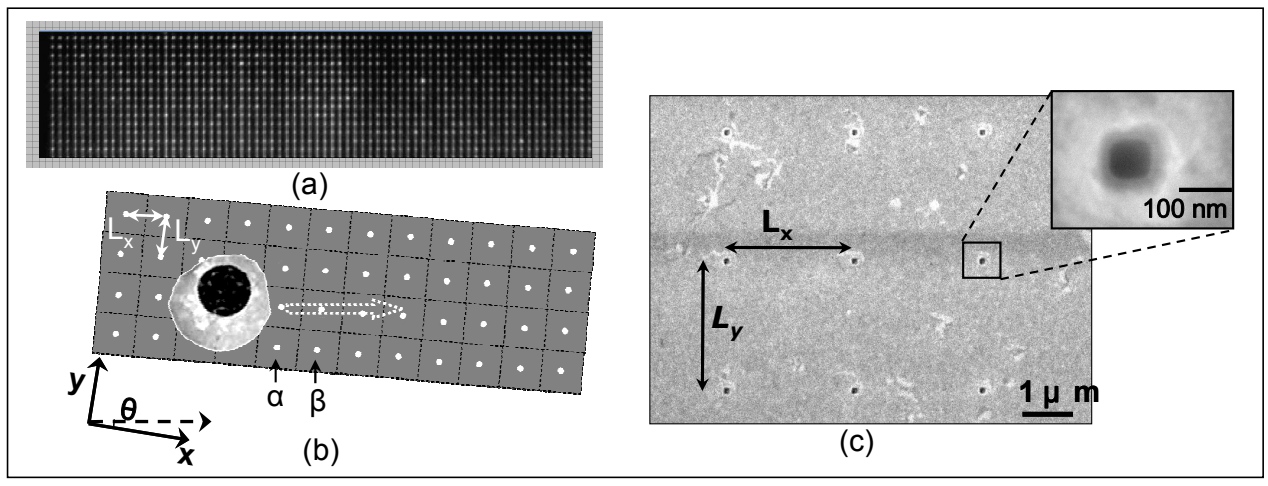

Figure 2. (a) The image of the 2D nanoaperture array shown on the recording CCD camera, when there is no sample above this region. (b) The orientation of the nanoaperture array with respect to the scanning direction of the sample. Lx: hole spacing in $x$ direction; Ly: hole spacing in y direction; $\theta$ : the angle between the scanning direction of the sample and $x$ axis. (c) Scanning electron microscope (SEM) image of the nanoaperture array. The substrate is aluminum. The inset shows one nanoaperture with a diameter about $90 \mathrm{~nm}$.

By knowing all the parameters mentioned in Eq. (1), the linear time-delay factors can be calculated and then used to remove this type of geometric distortion. For instance, when the 
sample is moving toward $+x$ direction (Fig. 2(b), the time delay $(\Delta t)$ between the [m, $\mathrm{n}]$ aperture and the $[1,1]$ aperture will be:

$$
\Delta t=\frac{\left.L_{x} \cos \theta\right)}{V}(n-1)-\frac{\left.L_{y} \sin \theta\right)}{V}(m-1)
$$

where $\mathrm{m}$ ( or $\mathrm{n}$ ) is the row (or column) index of the aperture. Similar expression can readily be drawn for the situation where the sample moves along $-\mathrm{x}$ direction.

During the fabrication of the nanoaperture array, a clean quartz wafer was coated with a $90 \mathrm{~nm}$ layer of aluminum by using an e-beam evaporator (CHA Mark 40). Then a thin layer of PMMA 950 was spun onto the aluminum plate; the 2D aperture array was patterned on PMMA by an e beam writer (Leica EBPG 5000) and then transferred onto aluminum by using a chlorine-based reactive ion etcher. The PMMA residue was then removed by acetone. In this experiment, the aperture size is $90 \mathrm{~nm}$ and the spacing between adjacent aperture is $2.5 \mu \mathrm{m}$ in both directions, i.e. $L_{x}=L_{y}=2.5 \mu \mathrm{m}$ (see Fig. 2(c)).

In the optical tweezer part, the speed of the trapped sample was controlled at $V=0.4$ $\mu \mathrm{m} / \mathrm{sec}$, and the camera's frame rate was $\delta t=0.125 \mathrm{~second} /$ frame. The skewing angle $(\theta)$ was fine adjusted to be 0.0154 in rad. Under this condition, the pixel size $\delta x$ was $\sim 50 \mathrm{~nm}$ and $\delta y$ was $\sim 40 \mathrm{~nm}$ which gave a theoretical resolution of $100 \mathrm{~nm}$ in $\mathrm{x}$ direction and $80 \mathrm{~nm}$ in $\mathrm{y}$ direction. Note that smaller pixel size can be accomplished by, for example, using a faster camera and smaller aperture spacing.

Besides pixel size, special attention has to be paid to the distance between the sample and the nanoaperture plane. It is well known that in order to accomplish super high resolution when using nanoapertures as imaging probes, the distance between the sample and the nanoaperture has to be much smaller than the illumination wavelength, i.e. $633 \mathrm{~nm}$ in this experiment. A rough estimate in our recent paper [9] shows that this distance (or more accurately, the depth of field) should be no more than the diameter of the nanoaperture. Therefore, we have to make sure that the optical tweezer, when moving the sample in the transverse plane, is not lifting the sample above the nanoaperture plane. Although optical tweezer as a powerful tool to manipulate microscopic objects has shown to be able to move the sample in vertical direction ( $\mathrm{z}$ direction) $[10,11]$, it is not capable of doing so in our setup.

We adopt the ray-optics method developed by Ashkin [12] to calculate the trapping force with $10 \mu \mathrm{m}$ polystyrene beads as an example. In our experiment, we used a $40 \mathrm{X}$ water immersion lens with a $\mathrm{NA}=0.80$. A polystyrene bead has a mass density $(\rho)$ about 1.05 , and refractive index at 1.57 at $633 \mathrm{~nm}$. The total power of the laser was $30 \mathrm{~mW}$ and the intensity distribution is assumed to be Gaussian. Since the polystyrene beads and the green algae we used were much larger than the wavelength of the trapping laser $(800 \mathrm{~nm})$, ray optics approximation is valid. The forces that tend to kick the microscopic object out of the laser trap include the balanced gravitation $\left(F_{\text {grav }}\right)$ of the object and the scattering force $\left(F_{S}\right)$ of the optical trap [12]. Both forces point to $-\mathrm{z}$ direction with the positive $\mathrm{z}$ direction defined according to the coordinate system shown in Fig. 2(b). $F_{\text {grav }}$ is $2.05 \mathrm{pN}$ and $F_{S}$ is calculated to be more than $8.2 \mathrm{pN}$. Thus, the summation of $F_{\text {grav }}$ and $F_{S}$ is more than $10.2 \mathrm{pN}$. On the other hand, the only force holding the sample within the optical trap is gradient force $\left(F_{G}\right)$ [12]. This force points to $+\mathrm{z}$ direction. In our setup, $F_{G}$ was no larger than $8.0 \mathrm{pN}$. Note that both $F_{G}$ and $F_{S}$ depend on the location of focal point inside the sample body, while $F_{\text {grav }}$ is independent of it. It is then clear that the polystyrene bead, although able to be translated in the transverse plane in our experiment, will not be lifted by the optical tweezer. This conclusion also holds for microspheres of other sizes. Note that biological samples will be even more difficult to lift because of their much lower refractive index contrast with respect to the surrounding water medium. However, the transverse scanning of green algae proves to be effective. 


\section{Results and discussion}

This novel OFM system was demonstrated by imaging polystyrene beads and green algae (Chlamydomonas) with the experimental conditions that were explained in Section 2. Both samples are mixed into a $0.2 \%$ bovine serum albumin (BSA) solution in order to reduce nonspecific bonding between the sample and the aluminum plate.

Fig. 3 (b, c) show two isolated polystyrene beads with a diameter of $10 \mu \mathrm{m}$ and $16 \mu \mathrm{m}$. Fig. 3 (b) shows fine structures inherent in the $10 \mu \mathrm{m}$ micro bead. It possibly stems from small surface roughness generated during fabrication. The near field scattering components from this roughness was propagated to far field and then captured by the CCD camera. Besides that, it is shown that there is a blurred profile surrounding the micro bead. This is probably due to the fact that although the center of the bead was close to the nanoaperture plane, the edge of it was about $5 \mu \mathrm{m}$ away from the nanoaperture plane, which means that the edge of the bead is far out of the depth of field of the nanoaperture array based imaging system. Same as conventional microscopy, falling out of depth of field would cause loss of resolution and blurring on the edge.

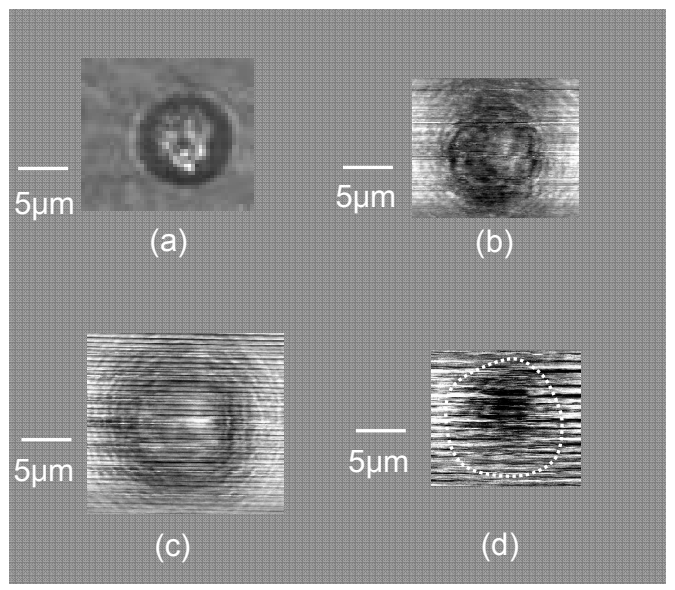

Figure 3: (a) Microscope image of a $10 \mu \mathrm{m}$ polystyrene bead. The image was taken by using the sample setup shown in Fig. 1(a) without the nanoaperture array chip. (b) OFM image of a $10 \mu \mathrm{m}$ bead (NIST standard); (c) OFM image of a $16 \mu \mathrm{m}$ bead; (d) OFM image of a green alga (Chlamydomonas). Image analysis is detailed in the text.

As can be seen in Fig. 3(c), there are discernable streaks running across the image in $\mathrm{x}$ direction. These streaks came from the power instability of the illumination He-Ne laser, as well as the diffraction patterns overshadowing the nanoaperture array. The diffraction pattern was probably caused by other samples floating in the water medium or imperfect alignment of some optical elements. The fluctuation of different apertures has low correlation and thus is extremely difficult to remove during data processing. Since Eq. (2) must be used to compensate for the time delay between adjacent apertures, the irreducible power fluctuation was elongated mostly in $\mathrm{x}$ direction and shows up in the figure. For the same reason, the Chlamydomonas image in Fig. 3(d) is also buried in the instability of the signals during the data acquisition. In this figure, the only discernible portion of the sample is probably its nucleus, which seems to have the best contrast under a transmission microscope. The edge of the sample is not easily recognizable and thus highlighted by a white dotted circle.

Nevertheless, the power instability issue could likely be solved by using high-intensity incoherent illumination source, such as LED array. DC-driven LED proves to be extremely 
stable. Our preliminary results have shown that nanoaperture array under the illumination of a LED array can be imaged onto the recording camera with a decent contrast.

\section{Conclusions}

In conclusion, we have demonstrated that optical tweezers can be combined with OFM for high resolution, high throughput imaging. In this study, nanoaperture array was used as fine illumination sources and we have rigorously justified that the conditions for ultra high resolution imaging were all satisfied during our experiment. Further verification of the imaging resolution is under way. Optical tweezers can readily be made into a tweezer array, which will dramatically improve the imaging efficiency. Additional imaging capability, e.g. fluorescence can also be added into the system.

\section{Acknowledgements}

We are grateful to Xiquan Cui, Zahid Yaqoob, Jigang Wu and Lap Man Lee from Caltech for their generous help. We thank UCLA's Nanolab and Caltech's nanofabrication group (Dr. Scherer) for their assistance in fabrication. This work was funded by DARPA Center for Optofluidic Integration (California Institute of Technology) and Coulter Foundation Career Award (funding)

\section{References and links}

1. W. L. Barnes, A. Dereux and T. W. Ebbesen, "Surface plasmon subwavelength optics," Nature 424, 824-830, (2003).

2. T. W. Ebbesen, H. J. Lezec, H. F. Ghaemi, T. Thio and P. A. Wolff, "Extraordinary optical transmission through sub-wavelength hole arrays," Nature 391, 667-669, (1998).

3. H. J. Lezec and T. Thio, "Diffracted evanescent wave model for enhanced and suppressed optical transmission through subwavelength hole arrays," Optics Express 12, 3629-3651, (2004).

4. D. E. Grupp, H. J. Lezec, T. Thio and T. W. Ebbesen, "Beyond the Bethe limit: Tunable enhanced light transmission through a single sub-wavelength aperture," Advanced Materials 11, 860-862, (1999).

5. E. H. Synge, "A suggested method for extending microscopic resolution into the ultramicroscopic region," Philosophical Magazine 6, 356-362, (1928).

6. B. Hecht, B. Sick, U. P. Wild, V. Deckert, R. Zenobi, O. J. F. Martin and D. W. Pohl, "Scanning near-field optical microscopy with aperture probes: Fundamentals and applications," Journal of Chemical Physics 112, 7761-7774, (2000).

7. D. Courjon, Near-field microscopy and near-field optics, London: Imperial College Press, 2003.

8. X. Heng, D. Erickson, L. R. Baugh, Z. Yaqoob, P. W. Sternberg, D. Psaltis and C. Yang, "Optofluidic microscopy- a method for implementing a high resolution optical microscope on a chip," Lab on a Chip 6, 1274 - 1276, (2006).

9. X. Heng, X. Q. Cui, D. W. Knapp, J. G. Wu, Z. Yaqoob, E. J. McDowell, D. Psaltis and C. H. Yang, "Characterization of light collection through a subwavelength aperture from a point source," Optics Express 14, 10410-10425, (2006). 
10. P. J. Rodrigo, I. R. Perch-Nielsen, C. A. Alonzo and J. Gluckstad, "GPC-based optical micromanipulation in 3D real-time using a single spatial light modulator," Optics Express 14, 13107-13112, (2006).

11. P. J. Rodrigo, V. R. Daria and J. Gluckstad, "Real-time interactive optical micromanipulation of a mixture of high- and low-index particles," Optics Express 12, 14171425, (2004).

12. A. Ashkin, "Forces of a Single-Beam Gradient Laser Trap on a Dielectric Sphere in the Ray Optics Regime," Biophys. J. 61, 569-582, (1992). 